\title{
On Conditional Decomposability
}

\author{
Jan Komenda ${ }^{\mathrm{a}}$, Tomáš Masopust ${ }^{\mathrm{a}, *}$, Jan H. van Schuppen ${ }^{\mathrm{b}}$ \\ ${ }^{a}$ Institute of Mathematics, Academy of Sciences of the Czech Republic, Žižkova 22, 61662 Brno, Czech Republic \\ ${ }^{b}$ CWI, P.O. Box 94079, 1090 GB Amsterdam, The Netherlands
}

\begin{abstract}
The requirement of a language to be conditionally decomposable is imposed on a specification language in the coordination supervisory control framework of discrete-event systems. In this paper, we present a polynomial-time algorithm for the verification whether a language is conditionally decomposable with respect to given alphabets. Moreover, we also present a polynomial-time algorithm to extend the common alphabet so that the language becomes conditionally decomposable. A relationship of conditional decomposability to nonblockingness of modular discrete-event systems is also discussed in this paper in the general settings. It is shown that conditional decomposability is a weaker condition than nonblockingness.
\end{abstract}

Keywords: Discrete-event system, coordination control, conditional decomposability.

2000 MSC: 93C65, 93A99, 93B50

\section{Introduction}

In the Ramadge-Wonham supervisory control framework, discrete-event systems are represented by deterministic finite automata. Given a specification language (usually also represented by a deterministic finite automaton), the aim of supervisory control is to construct a supervisor so that the closed-loop system satisfies the specification [1]. The theory is widely developed for the case where the system (plant) is monolithic. However, large engineering systems are typically constructed compositionally as a collection of many small components (subsystems) that are interconnected by rules; for instance, using a synchronous product or a communication protocol. This is especially true for discrete-event systems, where different local components run in parallel. Moreover, examples of supervisory control of modular discrete-event systems show that a coordinator is often necessary for achieving the required properties because the purely decentralized control architecture may fail in achieving these goals.

The notion of separability of a specification language has been introduced in [2], and says that a language $K$ over an alphabet $\bigcup_{i=1}^{n} E_{i}, n \geq 2$, is separable if $K=\|_{i=1}^{n} P_{i}(K)$, where for all $i=1,2, \ldots, n, P_{i}:\left(\bigcup E_{i}\right)^{*} \rightarrow E_{i}^{*}$ is a projection. A specification for a global system is separable if it can be represented (is fully determined) by local specifications for the component subsystems. It is very closely related to the notion of decomposability introduced in [3, 4] for decentralized discrete-event systems, which is also further studied in, e.g., [5]. Decomposability is a slightly more general condition because it involves not only the specification, but also the plant language, that is, a language $K \subseteq L$ over an alphabet $\bigcup_{i=1}^{n} E_{i}, n \geq 2$, is decomposable with respect to a plant language $L$ if $K=\left\|_{i=1}^{n} P_{i}(K)\right\| L$ : separability is then decomposability where $L=\left(\bigcup_{i=1}^{n} E_{i}\right)^{*}$ is the set of all strings over the global alphabet. In this paper, we slightly abuse the terminology and call a separable language in the sense of [2] also decomposable. It has been shown in [2] that decomposability is important because it is computationally cheaper to compute locally synthesized supervisors that constitute a solution of the supervisory control problem for this decomposable specification. Recently, the notion of decomposability has also been extended to automata as an automaton decomposability in, e.g., [6].

${ }^{*}$ Corresponding author. Institute of Mathematics, Academy of Sciences of the Czech Republic, Žižkova 22, 61662 Brno, Czech Republic, Tel. +420222090784, Fax. +420541218657

Email addresses: komenda@ipm.cz (Jan Komenda), masopust@math.cas.cz (Tomáš Masopust), J.H.van.Schuppen@cwi .nl (Jan H. van Schuppen) 
However, the assumption that a specification language is decomposable is too restrictive. Therefore, several authors have tried to find alternative techniques for general indecomposable specification languages; for instance, the approach of [7] based on partial controllability, which requires that all shared events are controllable, or the shared events must have the same controllability status (but then an additional condition of so-called mutual controllability [8] is needed).

In this paper, we study a weaker version of decomposability, so-called conditional decomposability, which has recently been introduced in [9] and studied in [10, 11] in the context of coordination supervisory control of discreteevent systems. It is defined as decomposability with respect to local alphabets augmented by the coordinator alphabet. The word conditional means that although a language is not decomposable with respect to the original local alphabets, it becomes decomposable with respect to the augmented ones, i.e., decomposability is only guaranteed (conditioned) by local event set extensions by coordinator events.

In the coordination control approach of modular discrete-event systems, the plant is formed as a parallel composition of two or more subsystems, while the specification language is represented over the global alphabet. Therefore, the property of conditional decomposability is required in this approach to distribute parts of the specification to the corresponding components to solve the problem locally. More specifically, we need to ensure that there exists a corresponding part of the specification for the coordinator and for each subsystem composed with the coordinator. Thus, if the specification is conditionally decomposable, we can take this decomposition as the corresponding parts for the subsystems composed with a coordinator and solve the problem locally.

Conditional decomposability depends on the alphabet of the coordinator, which can always be extended so that the specification is conditionally decomposable. In the worst (but unlikely) case all events must be put into the coordinator alphabet to make a language conditionally decomposable. But in the case when the coordinator alphabet would be too large it is better to divide the local subsystems into groups that are only loosely coupled and introduce several coordinators on smaller alphabets. In this paper, a polynomial-time algorithm is provided for the verification whether a language is conditionally decomposable. We make an important observation that the algorithm is linear in the number of local alphabets, while algorithms for checking similar properties (such as decomposability and coobservability) suffer from the exponential-time complexity with respect to the number of local alphabets. This algorithm is then modified so that it extends the coordinator alphabet to make the specification language conditionally decomposable. Furthermore, we discuss a relationship of conditional decomposability to nonblockingness of a coordinated system, where a coordinated system is understood as a modular system composed of two or more subsystems and a coordinator.

Finally, since one of the central notions of this paper is the notion of a (natural) projection, the reader is referred to [12] for more information on the state complexity of projected regular languages.

The rest of this paper is organized as follows. In Section 2, basic definitions and concepts of automata theory and discrete-event systems are recalled. In Section 3, a polynomial-time algorithm for testing conditional decomposability for a general monolithic system is presented. In Section 4 this algorithm is modified to extend the coordinator alphabet so that the specification becomes conditionally decomposable. In Section 5 , the relation of nonblockingness of a coordinated system with conditional decomposability is discussed. The conclusion with hints for future developments is presented in Section 6 .

\section{Preliminaries and definitions}

In this paper, we assume that the reader is familiar with the basic concepts of supervisory control theory [13] and automata theory [14]. For an alphabet $E$, defined as a finite nonempty set, $E^{*}$ denotes the free monoid generated by $E$, where the unit of $E^{*}$, the empty string, is denoted by $\varepsilon$. A language over $E$ is a subset of $E^{*}$. A prefix closure $\bar{L}$ of a language $L \subseteq E^{*}$ is the set of all prefixes of all words of $L$, i.e., it is defined as the set $\bar{L}=\left\{w \in E^{*} \mid \exists u \in E^{*}: w u \in L\right\}$. A language $L$ is said to be prefix-closed if $L=\bar{L}$.

In this paper, the notion of a generator is used to denote an incomplete deterministic finite automaton. A generator is a quintuple $G=\left(Q, E, \delta, q_{0}, F\right)$, where $Q$ is a finite set of states, $E$ is an input alphabet, $\delta: Q \times E \rightarrow Q$ is a partial transition function, $q_{0} \in Q$ is the initial state, and $F \subseteq Q$ is the set of final or marked states. In the usual way, $\delta$ is inductively extended to a function from $Q \times E^{*}$ to $Q$. The language generated by $G$ is defined as the set $L(G)=\left\{w \in E^{*} \mid \delta\left(q_{0}, w\right) \in Q\right\}$, and the language marked by $G$ is defined as the set $L_{m}(G)=\left\{w \in E^{*} \mid \delta\left(q_{0}, w\right) \in F\right\}$. Moreover, we use the predicate $\delta(q, a)$ ! to denote that the transition $\delta(q, a)$ is defined in state $q \in Q$ for event $a \in E$. 
For a generator $G$, let $\operatorname{trim}(G)$ denote the $\operatorname{trim}$ of $G$, that is, a generator $\operatorname{trim}(G)$ such that $\overline{L_{m}(\operatorname{trim}(G))}=$ $L(\operatorname{trim}(G))=\overline{L_{m}(G)}$. In other words, all reachable states of $G$ from which no marked state is reachable are removed (including the corresponding transitions), and only reachable states are considered in $\operatorname{trim}(G)$, see [13, 15]. A generator $G$ is said to be nonblocking if $\overline{L_{m}(G)}=L(G)$. Thus, $\operatorname{trim}(G)$ is always nonblocking.

A (natural) projection $P: E^{*} \rightarrow E_{0}^{*}$, where $E_{0} \subseteq E$ are alphabets, is a homomorphism defined so that $P(a)=\varepsilon$, for $a \in E \backslash E_{0}$, and $P(a)=a$, for $a \in E_{0}$. The inverse image of the projection $P$, denoted by $P^{-1}: E_{0}^{*} \rightarrow 2^{E^{*}}$, is defined so that for a language $L$ over the alphabet $E_{0}$, the set $P^{-1}(L)=\left\{s \in E^{*} \mid P(s) \in L\right\}$. In what follows, we use the notation $P_{j}^{i}$ to denote the projection from $E_{i}$ to $E_{j}$, that is, $P_{j}^{i}: E_{i}^{*} \rightarrow E_{j}^{*}$. In addition, we use the notation $E_{i+j}=E_{i} \cup E_{j}$, and, thus, $P_{k}^{i+j}$ denotes the projection from $E_{i+j}$ to $E_{k}$. If the projection is from the union of all the alphabets, then we simply use the notation $P_{i}:\left(\bigcup_{j} E_{j}\right)^{*} \rightarrow E_{i}^{*}$.

Let $L_{1} \subseteq E_{1}^{*}$ and $L_{2} \subseteq E_{2}^{*}$ be two languages. The parallel composition of $L_{1}$ and $L_{2}$ is defined as the language

$$
L_{1} \| L_{2}=P_{1}^{-1}\left(L_{1}\right) \cap P_{2}^{-1}\left(L_{2}\right),
$$

where $P_{1}:\left(E_{1} \cup E_{2}\right)^{*} \rightarrow E_{1}^{*}$ and $P_{2}:\left(E_{1} \cup E_{2}\right)^{*} \rightarrow E_{2}^{*}$. A similar definition in terms of generators follows. Let $G_{1}=\left(X_{1}, E_{1}, \delta_{1}, x_{01}, F_{1}\right)$ and $G_{2}=\left(X_{2}, E_{2}, \delta_{2}, x_{02}, F_{2}\right)$ be two generators. The parallel composition of $G_{1}$ and $G_{2}$ is the generator $G_{1} \| G_{2}$ defined as the accessible part of the generator $\left(X_{1} \times X_{2}, E_{1} \cup E_{2}, \delta,\left(x_{01}, x_{02}\right), F_{1} \times F_{2}\right)$, where

$$
\delta((x, y), e)= \begin{cases}\left(\delta_{1}(x, e), \delta_{2}(y, e)\right), & \text { if } \delta_{1}(x, e) ! \text { and } \delta_{2}(y, e) ! \\ \left(\delta_{1}(x, e), y\right), & \text { if } \delta_{1}(x, e) ! \text { and } e \notin E_{2} \\ \left(x, \delta_{2}(y, e)\right), & \text { if } e \notin E_{1} \text { and } \delta_{2}(y, e) ! \\ \text { undefined, } & \text { otherwise. }\end{cases}
$$

The automata definition is related to the language definition by the following properties: $L\left(G_{1} \| G_{2}\right)=L\left(G_{1}\right) \| L\left(G_{2}\right)$ and $L_{m}\left(G_{1} \| G_{2}\right)=L_{m}\left(G_{1}\right) \| L_{m}\left(G_{2}\right)$, see [13].

The automata-theoretic concept of nonblockingness of a composition of two generators $G_{1}$ and $G_{2}$ is equivalent to the language-theoretic concept of nonconflictness of two languages $L_{m}\left(G_{1}\right)$ and $L_{m}\left(G_{2}\right)$ if the generators $G_{1}$ and $G_{2}$ are nonblocking. Recall that two languages $L_{1}$ and $L_{2}$ are nonconflicting if $\overline{L_{1}} \| \overline{L_{2}}=\overline{L_{1} \| L_{2}}$, cf. [15, 16, 17].

Let $G$ be a generator and $P$ be a projection, then $P(G)$ denotes the minimal generator such that $L_{m}(P(G))=$ $P\left(L_{m}(G)\right)$ and $L(P(G))=P(L(G))$. For a construction of $P(G)$, the reader is referred to [13, 15].

Now, the main concept of interest of this paper, the concept of conditional decomposability, is defined. See also [9, 10, 11, 18] for the applications and further discussion concerning this concept.

Definition 1 (Conditional decomposability). A language $K$ over an alphabet $E_{1} \cup E_{2} \cup \ldots \cup E_{n}, n \geq 2$, is said to be conditionally decomposable with respect $E_{1}, E_{2}, \ldots, E_{n}$, and $E_{k}$, where $\bigcup_{i, j \in\{1,2, \ldots, n\}}^{i \neq j}\left(E_{i} \cap E_{j}\right) \subseteq E_{k} \subseteq \bigcup_{j=1}^{n} E_{j}$, if

$$
K=P_{1+k}(K)\left\|P_{2+k}(K)\right\| \ldots \| P_{n+k}(K) .
$$

Recall that $P_{i+k}$ denotes the projection from $\bigcup_{j=1}^{n} E_{j}$ to $E_{i+k}$.

Note that $\left\|_{i=1}^{n} P_{i+k}(K)=\left(\|_{i=1}^{n} P_{i+k}(K)\right)\right\| P_{k}(K)$ because $P_{i+k}(K) \subseteq\left(P_{k}^{i+k}\right)^{-1} P_{k}(K)$, which follows from the fact that $P_{k}^{i+k} P_{i+k}(K)=P_{k}(K)$. Hence, $\|_{i=1}^{n} P_{i+k}(K) \subseteq P_{k}^{-1} P_{k}(K)$. Moreover, if the language $K$ is given as a parallel composition of $n$ languages (over the required alphabets), then it is conditionally decomposable.

Lemma 2. A language $K \subseteq\left(E_{1} \cup E_{2} \cup \ldots \cup E_{n}\right)^{*}$ is conditionally decomposable with respect to alphabets $E_{1}, E_{2}, \ldots$, $E_{n}, E_{k}$ if and only if there exist languages $M_{i+k} \subseteq E_{i+k}^{*}, i=1,2, \ldots, n$, such that $K=\|_{i=1}^{n} M_{i+k}$.

Proof. If $K=\|_{i=1}^{n} P_{2+k}(K)$, define $M_{i+k}=P_{i+k}(K)$, for $i=1,2, \ldots, n$. On the other hand, assume that there exist languages $M_{i+k} \subseteq E_{i+k}^{*}, i=1,2, \ldots, n$, such that $K=\|_{i=1}^{n} M_{i+k}$. Obviously, $P_{i+k}(K) \subseteq M_{i+k}, i=1,2, \ldots, n$, which implies that $\|_{i=1}^{n} P_{i+k}(K) \subseteq K$. As it always holds that $K \subseteq P_{i+k}^{-1}\left[P_{i+k}(K)\right]$, the definition of the synchronous product implies that $K \subseteq \|_{i=1}^{n} P_{i+k}(K)$.

Note that $K=\|_{i=1}^{n} M_{i+k}$ implies that the languages $P_{i+k}(K) \subseteq M_{i+k}$, for $i=1,2, \ldots, n$, which means that $P_{i+k}(K)$ are the smallest languages whose parallel composition results in $K$. In other words, if $K$ is conditionally decomposable, then $P_{i+k}(K), i=1,2, \ldots, n$, is the smallest decomposition of $K$ with respect to the corresponding alphabets. 


\section{Polynomial Test of Conditional Decomposability}

In this section, we first construct a polynomial-time algorithm for the verification of conditional decomposability for alphabets $E_{1}, E_{2}$, and $E_{k}$, that is, for the case $n=2$, and then show how this is used to verify conditional decomposability for a general $n \geq 2$. To this end, consider a language $L$ over $E_{1} \cup E_{2}$, marked by a generator $G$. To verify whether or not $L$ is conditionally decomposable with respect $E_{1}, E_{2}$, and $E_{k}$, we construct a new structure as a parallel composition of two copies of $G$, denoted $f_{i+k}(G)$, for $i=1,2$, (see Example 3 and Figure 2) that simultaneously verifies that each word of $P_{1+k}(L) \| P_{2+k}(L)$ also belongs to $L=L_{m}(G) ; f_{i+k}(G)$ is constructed from the generator $G$ by renaming each event $e \in E_{j-k}=E_{j} \backslash E_{k}, j \neq i$, by a new event $\tilde{e} \in \tilde{E}_{j-k}$. In other words, each event $e$ which is not observed by $G$ according to the observable alphabet $E_{i} \cup E_{k}$ is replaced with a new event. Thus, the copy $f_{i+k}(G)$ is over the alphabet $E_{i+k} \cup \tilde{E}_{j-k}$, as demonstrated in the following example.

Example 3. Consider the language $L_{m}(G)$ marked by the generator $G$ depicted in Figure $\mathbb{\|}(a)$, where the corresponding alphabets are $E_{1}=\{a, b, d\}, E_{2}=\{a, c, d\}$, and $E_{k}=\{a, d\}$. The isomorphic generators $f_{1+k}(G)$ with renamed event $c$, and $f_{2+k}(G)$ with renamed event $b$ are depicted in Figure $\left.1 \mid b\right)$ and Figure $\|(\mid c)$ respectively.



(a) Generator $G$.

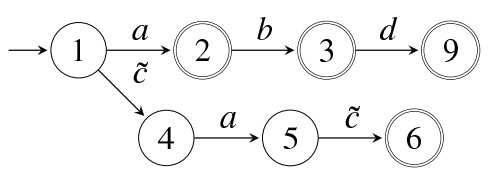

(b) Generator $f_{1+k}(G)$.

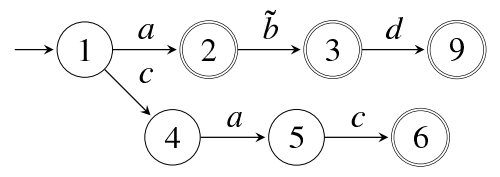

(c) Generator $f_{2+k}(G)$.

Figure 1: Generators $G, f_{1+k}(G)$ and $f_{2+k}(G)$.

More specifically, let $E_{1}, E_{2}, E_{k}$ be alphabets such that $E_{1} \cap E_{2} \subseteq E_{k} \subseteq E_{1} \cup E_{2}$, and define the global alphabet $E=E_{1} \cup E_{2}$. The structure is constructed as follows:

1. For the alphabet $E_{i} \backslash E_{k}$, where $i=1,2$, introduce a new alphabet $\tilde{E}_{i-k}=\left\{\tilde{a} \mid a \in E_{i} \backslash E_{k}\right\}$ that for each event $a \in E_{i} \backslash E_{k}$ contains a new event $\tilde{a}$. That is, $\tilde{E}_{i-k} \cap\left(E_{i} \backslash E_{k}\right)=\emptyset$ and there exists a bijection $g_{i-k}$ from $\left(E_{i} \backslash E_{k}\right)$ to $\tilde{E}_{i-k}$ such that $g_{i-k}(a)=\tilde{a}$. Note that $\tilde{E}_{1-k} \cap \tilde{E}_{2-k}=\emptyset$ because $E_{1} \cap E_{2} \subseteq E_{k}$.

2. Recall that $E_{i+k}=E_{i} \cup E_{k}$, for $i=1,2$, and let $\tilde{P}:\left(E \cup \tilde{E}_{1-k} \cup \tilde{E}_{2-k}\right)^{*} \rightarrow E^{*}$ be a projection.

3. Define two isomorphisms $f_{i+k}: E^{*} \rightarrow\left(E_{i+k} \cup \tilde{E}_{j-k}\right)^{*}$, where $i, j \in\{1,2\}, i \neq j$, so that

$$
f_{i+k}(a)= \begin{cases}a, & \text { for } a \in E_{i+k} \\ \tilde{a}, & \text { for } a \in E_{j} \backslash E_{k}\end{cases}
$$

Note that it immediately follows that $\left.\tilde{P}\left(f_{i+k}\left(L_{m}(G)\right)\right)=P_{i+k}\left(L_{m}(G)\right)\right)$ because both projections remove all events that are not in $E_{i+k}$.

4. For a generator $G=\left(Q, E, \delta, q_{0}, F\right)$, we abuse the notation and denote by $f_{i+k}(G)=\left(Q, E_{i+k} \cup \tilde{E}_{j-k}, \tilde{\delta}, q_{0}, F\right)$, where $j \neq i$, the generator isomorphic with $G$ where events are renamed according to the isomorphism $f_{i+k}$, and the transition function $\tilde{\delta}$ is define as $\tilde{\delta}\left(q, f_{i+k}(a)\right)=\delta(q, a)$.

5. Let $L \subseteq E^{*}$ be a language generated by a minimal generator $G$, and define the generator

$$
\tilde{G}=f_{1+k}(G) \| f_{2+k}(G)
$$

over the alphabet $E \cup \tilde{E}_{1-k} \cup \tilde{E}_{2-k}$. By the definition of $\tilde{G}$, the assumption that $E_{1} \cap E_{2} \subseteq E_{k}$ which ensures that $\tilde{P}$ distributes over the synchronous product (see Lemma 4 below), and Step 3 above, respectively, we have that

$$
\begin{aligned}
\tilde{P}\left(L_{m}(\tilde{G})\right) & =\tilde{P}\left(f_{1+k}\left(L_{m}(G)\right) \| f_{2+k}\left(L_{m}(G)\right)\right) \\
& =\tilde{P}\left(f_{1+k}\left(L_{m}(G)\right)\right) \| \tilde{P}\left(f_{2+k}\left(L_{m}(G)\right)\right) \\
& =P_{1+k}\left(L_{m}(G)\right) \| P_{2+k}\left(L_{m}(G)\right) .
\end{aligned}
$$


Lemma 4 ([15]). Let $E_{1} \cap E_{2} \subseteq E_{k} \subseteq E_{1} \cup E_{2}$, and let $L_{1} \subseteq E_{1}^{*}$ and $L_{2} \subseteq E_{2}^{*}$ be languages. Let $P_{k}: E^{*} \rightarrow E_{k}^{*}$ be a projection, then $P_{k}\left(L_{1} \| L_{2}\right)=P_{k}\left(L_{1}\right) \| P_{k}\left(L_{2}\right)$.

From the equations of (1), we immediately have the following result for conditional decomposability.

Theorem 5. The language $L_{m}(G)$ is conditionally decomposable with respect to alphabets $E_{1}, E_{2}, E_{k}$ if and only if it holds that $\tilde{P}\left(L_{m}(\tilde{G})\right)=L_{m}(G)$.

Proof. The proof follows immediately from the definition of conditional decomposability and (1).

However, the inclusion $\left.\left.L_{m}(G) \subseteq P_{1+k}\left(L_{m}(G)\right)\right) \| P_{2+k}\left(L_{m}(G)\right)\right)=\tilde{P}\left(L_{m}(\tilde{G})\right)$ always holds. Thus, only the opposite inclusion is of interest. This inclusion, $\tilde{P}\left(L_{m}(\tilde{G})\right) \subseteq L_{m}(G)$, holds if and only if $L_{m}(\tilde{G}) \subseteq \tilde{P}^{-1}\left(L_{m}(G)\right)$, which gives the following key theorem for testing conditional decomposability.

Theorem 6. The language $L_{m}(G)$ is conditionally decomposable with respect to alphabets $E_{1}, E_{2}, E_{k}$ if and only if the inclusion $L_{m}(\tilde{G}) \subseteq \tilde{P}^{-1}\left(L_{m}(G)\right)$ holds.

Proof. It remains to prove that $\tilde{P}\left(L_{m}(\tilde{G})\right) \subseteq L_{m}(G)$ if and only if $L_{m}(\tilde{G}) \subseteq \tilde{P}^{-1}\left(L_{m}(G)\right)$. However, if $\tilde{P}\left(L_{m}(\tilde{G})\right) \subseteq$ $L_{m}(G)$, then $L_{m}(\tilde{G}) \subseteq \tilde{P}^{-1} \tilde{P}\left(L_{m}(\tilde{G})\right) \subseteq \tilde{P}^{-1}\left(L_{m}(G)\right)$. On the other hand, assume that $L_{m}(\tilde{G}) \subseteq \tilde{P}^{-1}\left(L_{m}(G)\right)$. Then, $\tilde{P}\left(L_{m}(\tilde{G})\right) \subseteq \tilde{P} \tilde{P}^{-1}\left(L_{m}(G)\right)=L_{m}(G)$.

The verification of this inclusion results in Algorithm 1 for checking conditional decomposability of two components in polynomial time. Let a language $L$ be represented by the minimal generator $G=\left(Q, E, \delta, q_{0}, F\right)$ with the complete (total) transition function $\delta$ such that $L_{m}(G)=L$. If the transition function is not complete, the generator can be completed in time $O(|E| \cdot|Q|)$ by adding no more than one non-marked state and the missing transitions. Assume that the alphabets $E_{1}, E_{2}$, and $E_{k}$ are such that $E_{1} \cap E_{2} \subseteq E_{k} \subseteq E_{1} \cup E_{2}=E$, and see Algorithm 1 . To determine

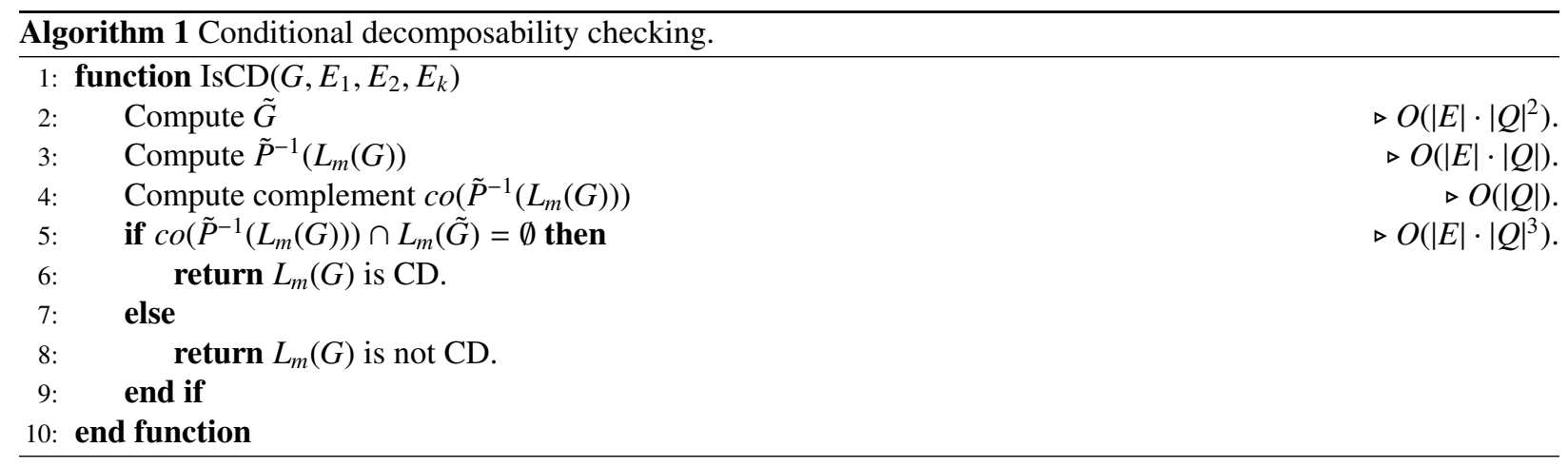

the time complexity of the algorithm, note that the computation is dominated by step 5, and thus the overall time complexity can be stated as $O\left(|E| \cdot|Q|^{3}\right)$. This also means that the space complexity is polynomial with respect to the number of states of the input generator $G$ because we do not need to use more space than $O\left(|E| \cdot|Q|^{3}\right)$. The complexity of individual steps of the algorithm are computed as follows. Step 2 is a parallel composition of two copies of $G$, which requires to create up to $|Q|^{2}$ states of the generator $\tilde{G}$, and for each of these states up to $|E|$ transitions. Step 3 requires up to $|E| \cdot|Q|$ steps because in each state, we have to add self-loops labeled by the new symbols from $\tilde{E}_{1-k} \cup \tilde{E}_{2-k}$. The complement in Step 4 is computed by interchanging the marking of states, cf. [19]. That is, marked states are unmarked and vice versa. As $G$ is complete, this results in a generator for the complement. Note that Steps 3 and 4 can be done at the same time. Finally, to decide the emptiness in Step 5 requires up to $|Q|^{2} \cdot|Q|$ using a standard product automaton, see [19], where for each state, up to $|E|$ transitions are constructed, and is verified by the reachability of a final state by the depth-first-search procedure in linear time [20]. Note also that it is a longstanding open problem whether the emptiness of intersection of two regular languages generated by generators with $m_{1}$ and $m_{2}$ states, respectively, can be decided in time $o\left(m_{1} \cdot m_{2}\right)$, cf. [21]. If this is possible, then the complexity of our algorithm can be improved accordingly.

We demonstrate our approach in the following example. 
Example 7. Consider the language $L_{m}(G)$ marked by the generator $G$ depicted in Figure 1 a where the corresponding alphabets are $E_{1}=\{a, b, d\}, E_{2}=\{a, c, d\}$, and $E_{k}=\{a, d\}$. The isomorphic generators $f_{1+k}(G)$ with renamed event $c$, and $f_{2+k}(G)$ with renamed event $b$ are depicted in Figure $1 \mid(b)$ and Figure 1$](c)$, respectively. Their parallel composition $\tilde{G}$ is shown in Figure 2. It is obvious that the string "cacb" belongs to the language $L_{m}(\tilde{G})$, whereas it does not belong to the language $\tilde{P}^{-1}\left(L_{m}(G)\right)$. Thus, by Theorem 6 the language $L_{m}(G)$ is not conditionally decomposable with respect to alphabets $E_{1}, E_{2}, E_{k}$.

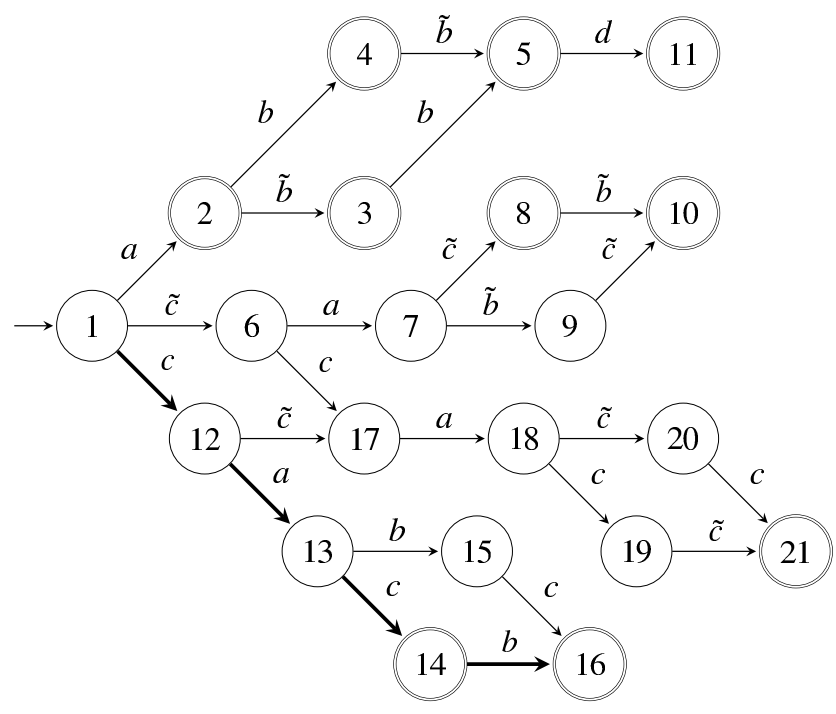

Figure 2: Generator $\tilde{G}=f_{1+k}(G) \| f_{2+k}(G)$ with a highlighted word violating conditional decomposability of the language $L_{m}(G)$.

Now, we generalize this approach to verifying conditional decomposability for a general number of $n \geq 2$ alphabets. The following theorem proves that we can directly use Algorithm 1 .

Theorem 8. Let $K$ be a language, and let $E_{i}$, for $i=1,2, \ldots, n, n \geq 2$, and $E_{k}$ be alphabets such that $\bigcup_{i \neq j}\left(E_{i} \cap E_{j}\right) \subseteq$ $E_{k} \subseteq \bigcup_{j=1}^{n} E_{j}$. Then, $P_{i+k}(K) \| P_{1+2+\ldots+(i-1)+(i+1)+\ldots+n+k}(K) \subseteq K$, for all $i=1,2, \ldots, n$, if and only if $K$ is conditionally decomposable with respect to alphabets $E_{i}, i=1,2, \ldots, n$, and $E_{k}$.

Proof. First, $P_{1+2+\ldots+(i-1)+(i+1)+\ldots+n+k}(K) \subseteq P_{1+k}(K)\left\|P_{2+k}(K)\right\| \ldots\left\|P_{(i-1)+k}(K)\right\| P_{(i+1)+k}(K)\|\ldots\| P_{n+k}(K)$ because for all $j \in\{1,2, \ldots, n\} \backslash\{i\}$, we have $P_{j+k}\left(P_{1+2+\ldots+(i-1)+(i+1)+\ldots+n+k}(K)\right)=P_{j+k}(K)$. Thus, if $K$ is conditionally decomposable, then $P_{i+k}(K)\left\|P_{1+2+\ldots+(i-1)+(i+1)+\ldots+n+k}(K) \subseteq P_{i+k}(K)\right\| P_{1+k}(K)\|\ldots\| P_{(i-1)+k}(K)\left\|P_{(i+1)+k}(K)\right\| \ldots \|$ $P_{n+k}(K)=K$, for all $i=1,2, \ldots, n$.

To prove the opposite implication, assume that $K$ is not conditionally decomposable. Then there exist $t_{i}=P_{i+k}\left(w_{i}\right)$, for some $w_{i} \in K$ and for all $i=1,2, \ldots, n$, such that $t_{1}\left\|t_{2}\right\| \ldots \| t_{n} \nsubseteq K$. We prove by induction on $i=1,2, \ldots, n-1$ that

$$
\left\{t_{i}\right\}\left\|\left\{t_{i-1}\right\}\right\| \ldots\left\|\left\{t_{2}\right\}\right\|\left\{t_{1}\right\} \| P_{(i+1)+(i+2)+\ldots+n+k}\left(w_{n}\right) \subseteq K .
$$

For $i=1$ and by the assumption, $\left\{t_{1}\right\}\left\|P_{2+3+\ldots+n+k}\left(w_{n}\right) \subseteq P_{1+k}(K)\right\| P_{2+3+\ldots+n+k}(K) \subseteq K$. Thus, we assume that it holds for all $i=1,2, \ldots, \ell, \ell<n-1$, and we prove it for $i=\ell+1$. By the induction hypothesis, $\left\{t_{\ell}\right\}\left\|\left\{t_{\ell-1}\right\}\right\| \ldots \|$ $\left\{t_{2}\right\}\left\|\left\{t_{1}\right\}\right\| P_{(\ell+1)+(\ell+2)+\ldots+n+k}\left(w_{n}\right) \subseteq K$. Then, using the projection $P_{1+2+\ldots+\ell+(\ell+2)+\ldots+n+k}$, we get that

$$
P_{1+2+\ldots+\ell+(\ell+2)+\ldots+n+k}\left(\left\{t_{\ell}\right\}\left\|\left\{t_{\ell-1}\right\}\right\| \ldots\left\|\left\{t_{2}\right\}\right\|\left\{t_{1}\right\} \| P_{(\ell+1)+(\ell+2)+\ldots+n+k}\left(w_{n}\right)\right) \subseteq P_{1+2+\ldots+\ell+(\ell+2)+\ldots+n+k}(K)
$$


and, by Lemma 4, we get that $P_{1+2+\ldots+\ell+(\ell+2)+\ldots+n+k}\left(\left\{t_{\ell}\right\}\left\|\left\{t_{\ell-1}\right\}\right\| \ldots\left\|\left\{t_{2}\right\}\right\|\left\{t_{1}\right\} \| P_{(\ell+1)+(\ell+2)+\ldots+n+k}\left(w_{n}\right)\right)=\left\{t_{\ell}\right\} \|$ $\left\{t_{\ell-1}\right\}\|\ldots\|\left\{t_{2}\right\} \Uparrow\left\{t_{1}\right\} \| P_{(\ell+2)+\ldots+n+k}\left(w_{n}\right)$. By this equality and the assumption for $i=\ell+1$, we have

$$
\begin{aligned}
& \left\{t_{\ell+1}\right\} \|\left[\left\{t_{\ell}\right\}\left\|\left\{t_{\ell-1}\right\}\right\| \ldots\left\|\left\{t_{2}\right\}\right\|\left\{t_{1}\right\} \| P_{(\ell+2)+\ldots+n+k}\left(w_{n}\right)\right] \\
& \subseteq P_{(\ell+1)+k}(K) \| P_{1+2+\ldots+\ell+(\ell+2)+\ldots+n+k}(K) \\
& \subseteq K
\end{aligned}
$$

as claimed. Then, substituting $i=n-1$ to 2 , we immediately have that $\left\{t_{n-1}\right\}\left\|\left\{t_{n-2}\right\}\right\| \ldots\left\|\left\{t_{2}\right\}\right\|\left\{t_{1}\right\} \| P_{n+k}\left(w_{n}\right) \subseteq K$, which together with $P_{n+k}\left(w_{n}\right)=t_{n}$ implies that $\left\{t_{n-1}\right\}\left\|\left\{t_{n-2}\right\}\right\| \ldots\left\|\left\{t_{2}\right\}\right\|\left\{t_{1}\right\} \|\left\{t_{n}\right\} \subseteq K$, which is a contradiction. Thus, $K$ is conditionally decomposable.

The previous theorem says that we can check conditional decomposability of a language $K$ by $n$ executions of Algorithm 1 . This means that the overall complexity of verifying conditional decomposability for a general number of alphabets, $n \geq 2$, is $O\left(n \cdot|E| \cdot|Q|^{3}\right)$, which is polynomial with respect to the number of states and the number of components.

To conclude this section, note that an example of an $r$-state automaton with $|E|=4$ and a projection reaching the exponential upper bound on the number of states, more precisely the upper bound $3 \cdot 2^{r-2}-1$, has been shown in [22]. Thus, the approach following the definition of conditional decomposability computing projections and parallel composition is exponential for that language even for the case of two alphabets. In comparison, the complexity of our algorithm is polynomial. A preliminary version of this algorithm has been implemented in libFAUDES [23].

\section{Extension of the coordinator alphabet}

According to Theorem 8 , we can again consider only the case $n=2$. To compute an extension of $E_{k}$ so that the language becomes conditionally decomposable, we modify Algorithm 1 to Algorithm 2, which uses more structural properties of the structure $\tilde{G}$. First, however, we explain the technique on an example.

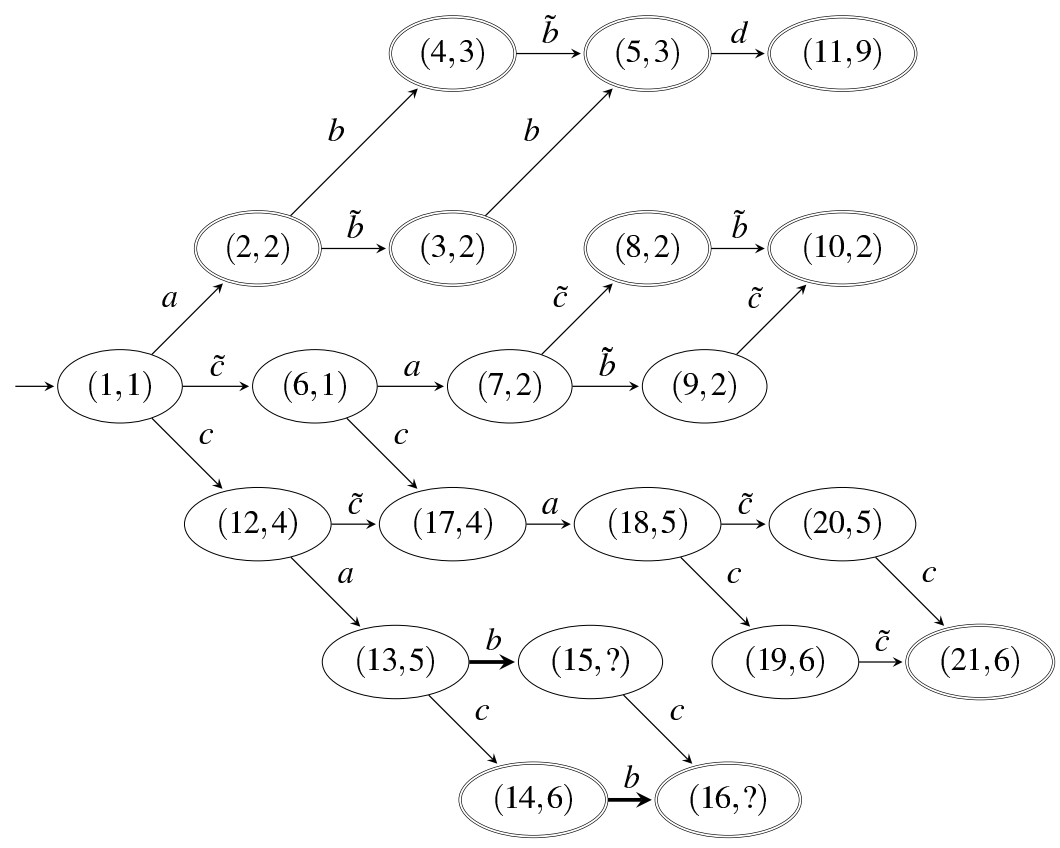

Figure 3: Generator $\tilde{G}$ with the corresponding states of $G \times \tilde{G}$. Note that transitions $\delta(5, b)$ and $\delta(6, b)$ are not defined in $G$, and, therefore, they violate conditional decomposability of the language $L_{m}(G)$. 
Example 9. Consider the generator $G$ and $\tilde{G}$ of Examples 3 and 7. The main idea of this technique is to construct, step-by-step, the parallel composition of $G$ and $\tilde{G}$, and to verify that all the steps possible in $\tilde{G}$ are also possible in $G$. In Figure $3 . \tilde{G}$ is extended with the states of $G$, written in the states of $\tilde{G}$. Note that after reading the string ca, the generator $\tilde{G}$ is in a state from which $b$ can be read, but $G$ being in state 5 can read only c. Because of this symbol $b$, the language $L_{m}(G)$ is not conditionally decomposable. The reader can verify that adding $b$ to $E_{k}$ results in the situation where $L_{m}(G)$ is conditionally decomposable with respect to $E_{1}, E_{2}$, and $E_{k} \cup\{b\}$.

Let a language $L$ be represented by the minimal generator $G=\left(Q, E, \delta, q_{0}, F\right)$ with the total transition function $\delta$ such that $L_{m}(G)=L$. Assume that alphabets $E_{1}, E_{2}, E_{k}$ satisfy $E_{1} \cap E_{2} \subseteq E_{k} \subseteq E_{1} \cup E_{2}=E$, and see Algorithm 2 . To prove that the algorithm is correct, note that it computes $L_{m}(\tilde{G}) \cap \tilde{P}^{-1}\left(L_{m}(G)\right)$ because $L_{m}(\tilde{G})=L_{m}(\operatorname{trim}(\tilde{G}))$. If

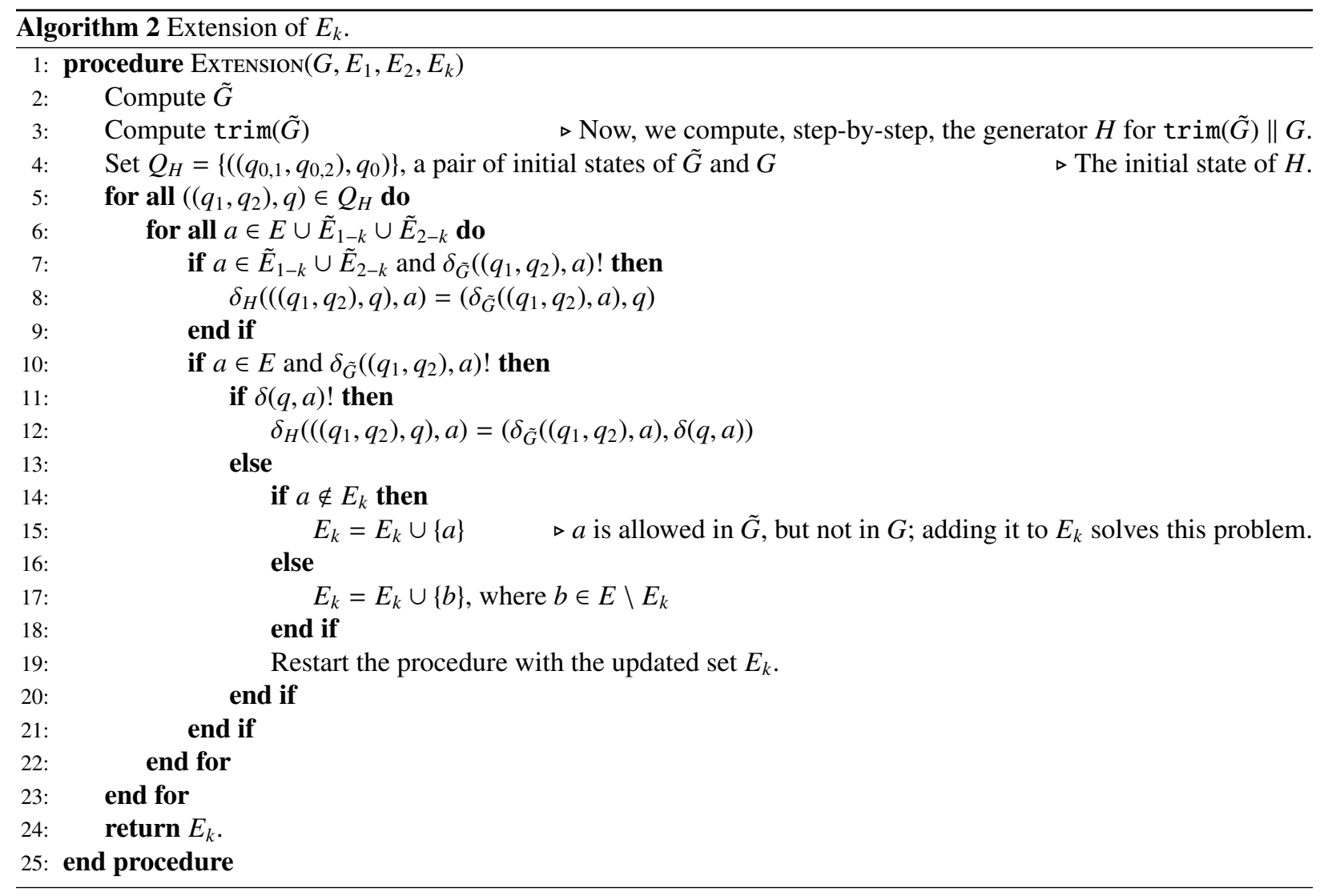

the condition on line 11 is always satisfied, it means that $L_{m}(\tilde{G}) \cap \tilde{P}^{-1}\left(L_{m}(G)\right)=L_{m}(\tilde{G})$. In other words, $L_{m}(\tilde{G}) \subseteq$ $\tilde{P}^{-1}\left(L_{m}(G)\right)$, which means by Theorem 6 that $L_{m}(G)$ is conditionally decomposable. On the other hand, if the condition on line 11 is not satisfied, there exists a string $s \in L(\operatorname{trim}(\tilde{G}))=\overline{L_{m}(\tilde{G})}$ such that $\tilde{P}(s) \notin L(G)=\overline{L_{m}(G)}$, where the last equality follows from the assumption that $G$ is minimal. This implies that $\tilde{P}\left(L_{m}(\tilde{G})\right) \nsubseteq L_{m}(G)$, hence $L_{m}(G)$ is not conditionally decomposable by Theorem 5 The algorithms halts because we have only a finite number of events to be added to $E_{k}$, and the language is conditionally decomposable for $E_{k}=E_{1} \cup E_{2}$.

The complexity of this algorithm is $O\left(|E|^{2} \cdot|Q|^{3}\right)$, which follows from the complexity of Algorithm 1 and the fact that, in the worst-case, we have to run the algorithm $|E|$ times. Note that the resulting extension depends on the order the states of $G$ and $\tilde{G}$ are examined. It should be clear that, in general, there might be different extensions (with respect to set inclusion) that correspond to different orders. This is a typical issue with algorithms extending the event sets in such a way that a particular property becomes true. There are examples where the algorithm does not construct the minimal possible extension. Note that to construct the minimal extension (with respect to set inclusion) is an NP-hard problem [24]. 
The following example demonstrates the situation where the event that causes the problem on line 11 already belongs to $E_{k}$. Thus, to solve the problem, another symbol from $E \backslash E_{k}$ must be added to $E_{k}$.

Example 10. Consider the generator $G$ depicted in Figure $4 \|$ a $)$ where the corresponding alphabets are $E_{1}=\left\{a_{1}, u\right\}$, $E_{2}=\left\{a_{2}, u\right\}$ and $E_{k}=\{u\}$. The isomorphic generators $f_{1+k}(G)$ with renamed event $a_{2}$ and $f_{2+k}(G)$ with renamed event $a_{1}$ are depicted in Figure $\left.4 \| b\right)$ and Figure $4 \|(c)$ respectively. The parallel composition $\tilde{G}=f_{1+k}(G) \| f_{2+k}(G)$ and one

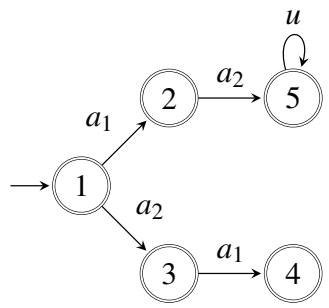

(a) Generator $G$.

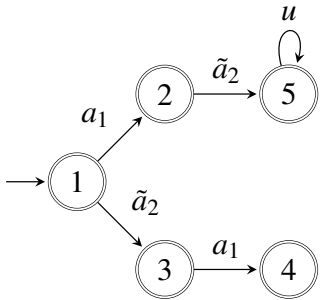

(b) Generator $f_{1+k}(G)$.

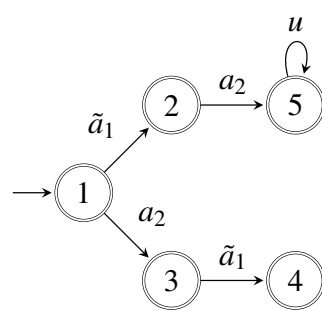

(c) Generator $f_{2+k}(G)$.

Figure 4: Generators $G, f_{1+k}(G)$ and $f_{2+k}(G)$.

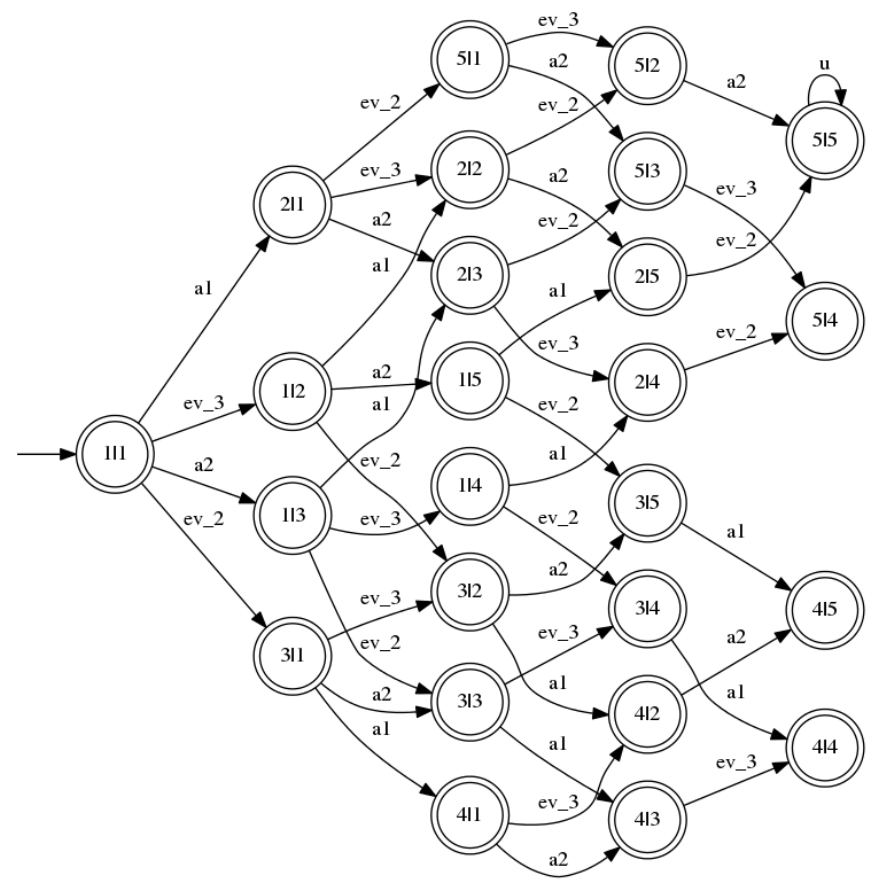

Figure 5: Generator $\tilde{G}$, where $E_{1}=\left\{u, a_{1}\right\}, E_{2}=\left\{u, a_{2}\right\}$, and $\tilde{a_{1}}=e v_{3}, \tilde{a_{2}}=e v_{2}$

can see that the string $\tilde{a}_{1} a_{2} a_{1} \tilde{a}_{2} u$ belongs to $L_{m}(\tilde{G})$, but does not belong to $\tilde{P}^{-1}\left(L_{m}(G)\right)$. By Theorem $5 . L_{m}(G)$ is not conditionally decomposable with respect to $E_{1}, E_{2}, E_{k}$. However, since u belongs to $E_{k}$, another event that does not belong to $E_{k}$ must be added to $E_{k}$. Namely, either $a_{1}$ or $a_{2}$.

This opens a field for potentially interesting heuristics. Indeed, to solve the problem on line 11, it does not make sense to add to $E_{k}$ an event that does not appear on the path leading to the problematic state. Thus, one could, for instance, store the last visited event from $E \backslash E_{k}$ that leads the generator $H=\tilde{G} \| G$ to a state where the problem was discovered. This event is then added to $E_{k}$ on line 17. 


\section{Relationship of nonblockingness of coordinated systems to conditional decomposability}

In this section, we study the relation between conditional decomposability and nonblockingness of coordinated discrete-event systems. A coordinated modular discrete-event system is a system composed (by parallel composition) of two or more subsystems. In this section, we consider the case of one central coordinator. Let $n \geq 2$, and let $G_{i}$, $i=1,2, \ldots, n$, be generators over the respective alphabets $E_{i}, i=1,2, \ldots, n$. The coordinated system $G$ is defined as $G=G_{1}\left\|G_{2}\right\| \ldots\left\|G_{n}\right\| G_{k}$, where $G_{k}$ is the coordinator over an alphabet $E_{k}$, which contains all shared events; namely, $E_{s} \subseteq E_{k}$, where $E_{s}$ is the set of all events that are shared by two or more components, defined as

$$
E_{s}=\bigcup_{i, j \in\{1, \ldots, n\}}^{i \neq j}\left(E_{i} \cap E_{j}\right) .
$$

This is a standard assumption in hierarchical decentralized control where the coordinator level plays a role of the high (abstracted) level of hierarchical control.

In the following theorem, we show the relation between nonblockingness of a coordinated system and conditional decomposability of that system. First, however, we need the following auxiliary lemmas.

Lemma 11 (Proposition 4.1 in [16]). Let $L \subseteq E^{*}$ be a language and $P_{k}: E^{*} \rightarrow E_{k}^{*}$ be a projection with $E_{k} \subseteq E$, for some alphabet $E$. Then, $P_{k}(\bar{L})=\overline{P_{k}(L)}$.

Lemma 12. Let $E$ be an alphabet, $L \subseteq E^{*}$ be a language, and $P_{k}: E^{*} \rightarrow E_{k}^{*}$ be a projection with $E_{k} \subseteq E$, for some alphabet $E$. Then, $L \| P_{k}(L)=L$.

Proof. By definition, $L \| P_{k}(L)=L \cap P_{k}^{-1} P_{k}(L)$, and it is not hard to see that $L \subseteq P_{k}^{-1} P_{k}(L)$.

Theorem 13. Let $n \geq 2$, and let $G_{i}$, for $i=1,2, \ldots, n$, be generators over the alphabets $E_{i}, i=1,2, \ldots, n$, respectively. Let $G_{k}$ be a generator over an alphabet $E_{k}$ such that $E_{s} \subseteq E_{k} \subseteq \bigcup_{i=1}^{n} E_{i}$. Then, the coordinated system $G=G_{1}\left\|G_{2}\right\| \ldots\left\|G_{n}\right\| G_{k}$ is nonblocking if and only if the following conditions both hold:

1. $G_{i}\left\|G_{k}\right\| \|_{j \neq i} P_{k}\left(G_{j}\right)$, for all $i=1,2, \ldots, n$, are nonblocking and

2. $\overline{L_{m}(G)}$ is conditionally decomposable with respect to the alphabets $E_{1}, E_{2}, \ldots, E_{n}, E_{k}$.

Proof. The following always holds for all $i=1,2, \ldots, n, n \geq 2$ :

$$
\begin{aligned}
\overline{L_{m}(G)} \subseteq & P_{1+k}\left(\overline{L_{m}(G)}\right)\|\ldots\| P_{n+k}\left(\overline{L_{m}(G)}\right) \\
\subseteq & P_{1+k}(L(G))\|\ldots\| P_{n+k}(L(G)) \\
= & L\left(G_{1}\left\|G_{k}\right\| P_{k}\left(G_{2}\left\|G_{3}\right\| \ldots \| G_{n}\right)\right) \\
& \| L\left(G_{2}\left\|G_{k}\right\| P_{k}\left(G_{1}\left\|G_{3}\right\| \ldots \| G_{n}\right)\right) \\
& \vdots \\
& \| L\left(G_{n}\left\|G_{k}\right\| P_{k}\left(G_{1}\left\|G_{2}\right\| \ldots \| G_{n-1}\right)\right) \\
= & L(G),
\end{aligned}
$$

where the last equation follows from the idempotent property of the parallel composition and Lemma 12 If the language $\overline{L_{m}(G)}$ is nonblocking, then the inclusions become equalities. Thus, from the first equality, we get that the language $\overline{L_{m}(G)}$ is conditionally decomposable as required in item 2 of the theorem. Similarly, for all $i=1,2, \ldots, n$,

$$
\begin{aligned}
P_{i+k}\left(\overline{L_{m}(G)}\right) & =\overline{P_{i+k}\left(L_{m}(G)\right)}=\overline{L_{m}\left(G_{i} \| G_{k}\right) \| P_{i+k}\left(L_{m}\left(\|_{j \neq i} G_{j}\right)\right)} \\
& =\overline{L_{m}\left(G_{i}\left\|G_{k}\right\| P_{i+k}\left(\|_{j \neq i} G_{j}\right)\right.} \\
& \subseteq \overline{L_{m}\left(G_{i}\right)}\left\|\overline{L_{m}\left(G_{k}\right)}\right\| \overline{P_{i+k}\left(\|_{j \neq i} L_{m}\left(G_{j}\right)\right)} \\
& \subseteq L\left(G_{i}\right)\left\|L\left(G_{k}\right)\right\| P_{i+k}\left(\|_{j \neq i} L\left(G_{j}\right)\right) \\
& =P_{i+k}(L(G)),
\end{aligned}
$$


where the first equality holds by Lemma 11 , the second equality holds by Lemma 4 because we project to the alphabet $E_{i} \cup E_{k}$ that includes the intersection of $E_{i} \cup E_{k}$ and $\bigcup_{j \neq i} E_{j}$, namely $E_{k}$. Finally, the last equality holds by the same argument as the second equality. Hence, if the global plant is nonblocking, the inclusions become equalities, which means that the subsystems $G_{i}\left\|G_{k}\right\| P_{i+k}\left(\|_{j \neq i} G_{j}\right)=G_{i}\left\|G_{k}\right\| \|_{j \neq i} P_{k}\left(G_{j}\right)$ are nonblocking.

On the other hand, from the assumptions 1 and 2 we immediately get that both inclusions in (3) are equalities. Thus, the implication holds.

Note that Condition 2 of Theorem 13 does not hold in general because one inclusion of conditional decomposability, namely $\overline{L_{m}(G)} \subseteq P_{1+k}\left(\overline{L_{m}(G)}\right) \| P_{2+k}\left(\overline{L_{m}(G)}\right)$, can be strict. Thus, the prefix closure of the marked language $\overline{L_{m}\left(G_{1}\left\|G_{2}\right\| G_{k}\right)}$ of the coordinated system consisting of subsystems $G_{1}$ and $G_{2}$ and a coordinator $G_{k}$ is not in general conditionally decomposable with respect to alphabets $E_{1}, E_{2}, E_{k}$ as demonstrated in the following example.

Example 14. Consider two subsystems $G_{1}$ and $G_{2}$, and a coordinator $G_{k}$ as depicted in Figure 6, where the corresponding alphabets are $E_{1}=\{a, b, d\}, E_{2}=\{a, c, d\}$, and $E_{k}=\{a, d\}$. Then, we can consider the string cacb

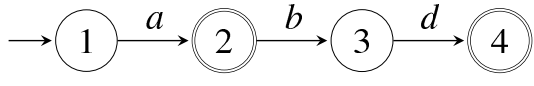

(a) Generator $G_{1}$.

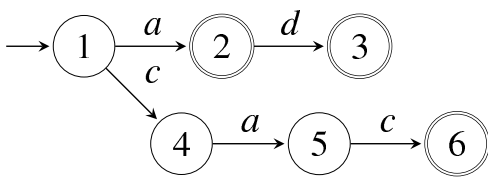

(c) Generator $G_{2}$.

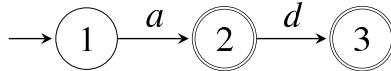

(b) Generator $G_{k}$

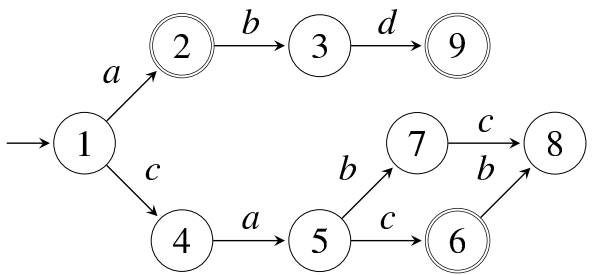

(d) Generator $G_{1}\left\|G_{2}\right\| G_{k}$.

Figure 6: Generators $G_{1}, G_{2}, G_{k}$, and $G_{1}\left\|G_{2}\right\| G_{k}$.

and see that its projection $P_{1+k}(c a c b)=$ ab belongs to the language $P_{1+k}\left(\overline{L_{m}\left(G_{1}\left\|G_{2}\right\| G_{k}\right)}\right)$, and the projection $P_{2+k}(c a c b)=$ cac belongs to the language $P_{2+k}\left(\overline{L_{m}\left(G_{1}\left\|G_{2}\right\| G_{k}\right)}\right)$. However, this means that the string cacb belongs to the composition $P_{1+k}\left(\overline{L_{m}\left(G_{1}\left\|G_{2}\right\| G_{k}\right)}\right) \| P_{1+k}\left(\overline{L_{m}\left(G_{1}\left\|G_{2}\right\| G_{k}\right)}\right)$. On the other hand, the string cacb is not a prefix of any string belonging to the marked language $\overline{L_{m}\left(G_{1}\left\|G_{2}\right\| G_{k}\right)}$ of the coordinated system as is easily seen in Figure $G(d)$ Thus, the language is not conditionally decomposable with respect to alphabets $E_{1}, E_{2}, E_{k}$.

Note that it follows from (3) that conditional decomposability is a weaker condition than nonblockingness. This is because conditional decomposability requires only the first inclusion to be equality, while nonblockingness requires both the inclusions to be equalities. The fundamental question is whether it is possible to decide in a distributed way without computing the whole plant whether $\overline{L_{m}\left(\left\|_{i=1}^{n} G_{i}\right\| G_{k}\right)}$ is conditionally decomposable. The algorithm described in the previous section requires the computation of the whole plant.

A specific choice of $L_{m}\left(G_{k}\right) \subseteq \bigcap_{i=1}^{n} P_{k}\left(L_{m}\left(G_{i}\right)\right)$, respectively $L_{m}\left(G_{k}\right)=\bigcap_{i=1}^{n} P_{k}\left(L_{m}\left(G_{i}\right)\right)$, yields Corollaries 15 and 16 below, respectively.

Corollary 15. Let $G_{1}, G_{2}, \ldots, G_{n}, G_{k}$ be nonblocking generators over the alphabets $E_{1}, E_{2}, \ldots, E_{n}, E_{k}$, respectively, such that $E_{s} \subseteq E_{k} \subseteq \bigcup_{i=1}^{n} E_{i}$. Assume that $L_{m}\left(G_{k}\right) \subseteq \bigcap_{i=1}^{n} P_{k}\left(L_{m}\left(G_{i}\right)\right)$. Then, the coordinated system $G=G_{1}\left\|G_{2}\right\|$ $\ldots\left\|G_{n}\right\| G_{k}$ is nonblocking if and only if the following conditions both hold:

1. $G_{i} \| G_{k}$ are nonblocking, for all $i=1,2, \ldots, n$, and

2. $\overline{L_{m}(G)}$ is conditionally decomposable with respect to the alphabets $E_{1}, E_{2}, \ldots, E_{n}, E_{k}$.

Proof. By the assumption, $L_{m}\left(G_{k}\right) \subseteq \bigcap_{i} P_{k}\left(L_{m}\left(G_{i}\right)\right)$. Applying the prefix closure to the previous inclusion results in the inclusion $\left.L\left(G_{k}\right)=\overline{L_{m}\left(G_{k}\right)} \subseteq \overline{P_{k}\left(\bigcap_{i} L_{m}\left(G_{i}\right)\right)} \subseteq \bigcap_{i} P_{k} \overline{\left(L_{m}\left(G_{i}\right)\right.}\right)=\bigcap_{i} P_{k}\left(L\left(G_{i}\right)\right)=\|_{i} P_{k}\left(L\left(G_{i}\right)\right)$. From this, it follows that $L\left(G_{k}\right) \| P_{k}\left(L\left(G_{i}\right)\right)=L\left(G_{k}\right)$, for $i=1,2, \ldots, n$, which implies that $G_{i}\left\|G_{k}\right\|\left\|_{j \neq i} P_{k}\left(G_{j}\right)=G_{i}\right\| G_{k}$. Thus, item 1 of Theorem 13 reduces to item 1 of this corollary. 
Corollary 16. Let $G_{1}, G_{2}, \ldots, G_{n}, G_{k}$ be nonblocking generators over the alphabets $E_{1}, E_{2}, \ldots, E_{n}, E_{k}$, respectively, such that $E_{s} \subseteq E_{k} \subseteq \bigcup_{i=1}^{n} E_{i}$, and assume that $L_{m}\left(G_{k}\right)=\bigcap_{i=1}^{n} P_{k}\left(L_{m}\left(G_{i}\right)\right)$. Then, the coordinated system $G=\left\|_{i=1}^{n} G_{i}\right\|$ $G_{k}$ is nonblocking if and only if the following conditions both hold:

1. $G_{i} \| G_{k}$ are nonblocking, for all $i=1,2, \ldots, n$, and

2. $\overline{L_{m}\left(G_{1}\left\|G_{2}\right\| \ldots \| G_{n}\right)}$ is conditionally decomposable with respect to alphabets $E_{1}, E_{2}, \ldots, E_{n}, E_{k}$.

Proof. The proof follows immediately from the previous corollary and the fact that $\left\|_{i} L_{m}\left(G_{i}\right)\right\| L_{m}\left(G_{k}\right)=\left(\|\|_{i} L_{m}\left(G_{i}\right)\right) \|$ $\left(\|_{i} P_{k}\left(L_{m}\left(G_{i}\right)\right)\right)$, which is equal to $\|_{i} L_{m}\left(G_{i}\right)$ by Lemma 12 , which reduces item 2 of Corollary 15 to the form of item 2 of this corollary.

The last corollary is particularly interesting because the coordinated modular discrete-event system coincides with the original plant and, therefore, nonblockingness of the original plant itself can be checked using the approach based on a coordinator, provided that we can verify item 2 in a distributed way.

The approach discussed above is based on projections, and the only known sufficient condition ensuring that the projected automaton is smaller with respect to the number of states than the original one is the observer property mentioned below. This topic requires further investigation because the observer property is only a sufficient condition, not necessary; there are examples of projected automata that are smaller than original automata without the projections satisfying the observer property. For completeness, however, we now discuss the case of projections satisfying the observer property and show that it corresponds to the known results discussed in [16] and in references therein.

Finally, we mention that in practice one central coordinator is particularly useful for loosely coupled subsystems, where the interaction between the subsystems (via synchronisation) is not too strong. Otherwise, a general multilevel hierarchy approach should be adopted, where the subsystems are aggregated into groups that are only loosely coupled. This is, however, very technical and left for a future study.

\subsection{Observer property}

The previous results are of interest in the case the projected systems $P_{k}\left(G_{i}\right)$, for $i=1,2, \ldots, n$, are significantly smaller than the original systems $G_{i}$. So far, the only known condition ensuring this is a so-called observer property.

Definition 17 (Observer property). Let $E_{k} \subseteq E$ be alphabets. A projection $P_{k}: E^{*} \rightarrow E_{k}^{*}$ is an $L$-observer for a language $L \subseteq E^{*}$ if the following holds: for all strings $t \in P(L)$ and $s \in \bar{L}$, if $P(s)$ is a prefix of $t$, then there exists $u \in E^{*}$ such that $s u \in L$ and $P(s u)=t$.

The following lemma proves that if the projections are observers, then item 2 of the previous results can be eliminated because it is always satisfied.

Lemma 18. Let $G_{i}, 1,2, \ldots, n, n \geq 2$, and $G_{k}$ be generators over the alphabets $E_{i}, i=1,2, \ldots, n$, and $E_{k}$, respectively, such that $E_{s} \subseteq E_{k} \subseteq \bigcup_{i} E_{i}$, and denote $G=\left\|_{i} G_{i}\right\| G_{k}$. If the projections $P_{k}^{i+k}$ are $P_{i+k}\left(L_{m}(G)\right)$-observers, for $i=1,2, \ldots, n$, then the language $\overline{L_{m}(G)}$ is conditionally decomposable with respect to $E_{i}, i=1,2, \ldots, n$, and $E_{k}$.

Proof. By Lemma 11, showing the first equality, it holds in general that

$$
\begin{aligned}
& \left\|_{i=1}^{n} P_{i+k}\left(\overline{L_{m}(G)}\right)=\right\|_{i=1}^{n} \overline{P_{i+k}\left(L_{m}(G)\right)} \supseteq \overline{\|_{i=1}^{n} P_{i+k}\left(L_{m}(G)\right)} \\
& =\overline{\|_{i=1}^{n} L_{m}\left(G_{i}\left\|G_{k}\right\| \|_{j \neq i} P_{k}\left(G_{j}\right)\right)}=\overline{L_{m}(G)} .
\end{aligned}
$$

The last equality follows from the commutativity of the synchronous product and Lemma 12, By [25], it holds that $\|_{i=1}^{n} \overline{P_{i+k}\left(L_{m}(G)\right)}=\overline{\|_{i=1}^{n} P_{i+k}\left(L_{m}(G)\right)}$ if and only if $\|_{i=1}^{n} \overline{P_{k}\left(L_{m}(G)\right)}=\overline{\|_{i=1}^{n} P_{k}\left(L_{m}(G)\right)}$, and the later equality is obviously satisfied. Thus, the former equality implies by 44 that the language $\overline{L_{m}(G)}$ is conditionally decomposable with respect to alphabets $E_{1}, E_{2}, E_{k}$, which was to be shown. 
As mentioned in the previous proof, when we consider all the assumptions, Feng [16] (see also the references therein) has shown that if the projection $P_{k}$ is an observer for $L_{1}$ and $L_{2}$, then $L_{1} \| L_{2}$ is nonconflicting if and only if $P_{k}\left(L_{1}\right) \| P_{k}\left(L_{2}\right)$ is nonconflicting. This is generalized to arbitrary components in [25]. Note that using this property on item 1 of Corollary 16, together with the previous lemma and the fact that the observers preserve parallel composition, [25], results in the following corollary, which generalizes the results shown in [16] for two components.

Corollary 19. Let $G_{i}, 1,2, \ldots, n, n \geq 2$, and $G_{k}$ be nonblocking generators over the alphabets $E_{i}, i=1,2, \ldots, n$, and $E_{k}$, respectively, such that $E_{s} \subseteq E_{k} \subseteq \bigcup_{i} E_{i}$, and assume that $L_{m}\left(G_{k}\right)=\bigcap_{i} P_{k}\left(L_{m}\left(G_{i}\right)\right)$ and $L\left(G_{k}\right)=\bigcap_{i} P_{k}\left(L\left(G_{i}\right)\right)$. Assume that the projections $P_{k}^{i}$ are $L_{m}\left(G_{i}\right)$-observers, for $i=1,2, \ldots, n$. Then, the coordinated system $\left\|_{i} G_{i}\right\| G_{k}$ is nonblocking if and only if $G_{k}$ is nonblocking.

This works because the projection is an observer. However, there are languages which are conditionally decomposable, but the projections from Lemma 18 are not observers. For instance, consider a language $L=\{b a, c d b, d c b\}$. It can be verified that $L$ is conditionally decomposable with respect to the alphabets $E_{1}=\{a, b, c\}, E_{2}=\{a, b, d\}$, and $E_{k}=\{a, b\}$, and that the projections $P_{k}^{i+k}$ are not $P_{i+k}(L)$-observers, for $i=1,2$. Note that $P_{1+k}(L)=\{b a, c b\}$ and $P_{2+k}(L)=\{b a, d b\}$. Then, for $t=b$ and $s=c b$ (for $i=1$, or $s=d b$ for $i=2$ ), there is no extension of $c b$ such that

$P_{k}^{1+k}(c b)=b a$. Hence, the projections are not observers. For that reason, we consider in this paper a more general assumption that the projections are such that the projected generators are smaller than the original generators. Note that the conditions under which this is true still need to be investigated. Finally, note that for the verification whether the subsystems $G_{i}\left\|G_{k}\right\| \|_{j \neq i} P_{k}\left(G_{j}\right)$ are nonblocking, the methods presented in [26, 27] can be used, combined with further usage of Binary Decision Diagrams [28] or state-tree structures [29] to perform the calculations.

\section{Conclusion}

The main contributions of this paper are polynomial-time algorithms for the verification whether a language is conditionally decomposable and for an extension of the coordinator alphabet $E_{k}$. Our approach to extend the alphabet $E_{k}$ is based on the successive addition of events to the alphabet $E_{k}$. Another approach has recently been discussed in [30], where the problematic transitions are identified, and the events of these transitions are renamed. From the viewpoint of applications, however, our approach can directly be used in coordination control for which it has primarily been developed. On the other hand, the approach from [30] has so far no direct applications in the coordination control framework, which is under investigation. Nevertheless, the algorithms presented here can also be used for the approach presented in [30].

Particularly valuable is the property that algorithms for checking conditional decomposability of a language with respect to alphabets is linear in the number of alphabets (that corresponds to local controllers in coordination control). No such results are known for co-observability (the notion playing a central role in decentralized control) and the related property of decomposability. It is well-known that co-observability is equivalent to decomposability under some reasonable assumptions on locally controllable and locally observable alphabets. Since conditional decomposability can be seen as decomposability with respect to particular alphabets (enriched by the coordinator events), it appears that our results about conditional decomposability will have impact on decentralized control with communicating supervisors. Indeed, co-observability is ensured by a special types of communication (which corresponds to enriching the sets of locally observable events such that a specification language becomes co-observable) in a similar way as decomposability is imposed by enriching the alphabets of local supervisors.

The paper also compares the property of conditional decomposability to nonblockingness of a coordinated system. The current low complexity tests of practical interest are based on the observer property because it is the only known condition ensuring that the projected generator is smaller than the original one. However, this is only a sufficient condition and further investigation is needed. It is our plan to further investigate the construction procedures for designing coordinators for nonblockingness that are as small as possible and we will combine these results with those obtained in coordination control for safety so that both nonblockingness and safety issues can be efficiently handled using coordination control. 


\section{Acknowledgments}

The authors gratefully acknowledge very useful suggestions and comments of the anonymous referees. The research has been supported by the GAČR grants P103/11/0517 and P202/11/P028, and by RVO: 67985840 .

\section{References}

[1] P. J. Ramadge, W. M. Wonham, Supervisory control of a class of discrete event processes, SIAM J. Control Optim. 25 (1987) $206-230$.

[2] Y. Willner, M. Heymann, Supervisory control of concurrent discrete-event systems, Internat. J. Control 54 (1991) 1143-1169.

[3] K. Rudie, W. Wonham, Supervisory control of communicating processes, in: Proc. of International Symposium on Protocol Specification, Testing and Verification X, Elsevier Science Publishers, Amsterdam, The Netherlands, 1990, pp. 243-257.

[4] K. Rudie, W. M. Wonham, Think globally, act locally: Decentralized supervisory control, IEEE Trans. Automat. Control 37 (1992) $1692-$ 1708.

[5] S. Jiang, R. Kumar, Decentralized control of discrete event systems with specializations to local control and concurrent systems, IEEE Trans. Syst. Man Cybern. B 30 (2000) 653-660.

[6] M. Karimadini, H. Lin, Decomposability of global tasks for multi-agent systems, in: Proc. of CDC 2010, pp. 4192-4197.

[7] B. Gaudin, H. Marchand, An efficient modular method for the control of concurrent discrete event systems: A language-based approach, Discrete Event Dyn. Syst. 17 (2007) 179-209.

[8] J. Komenda, J. H. van Schuppen, B. Gaudin, H. Marchand, Supervisory control of modular systems with global specification languages, Automatica 44 (2008) 1127-1134.

[9] J. Komenda, J. H. van Schuppen, Coordination control of discrete event systems, in: Proc. of WODES 2008, pp. 9-15.

[10] J. Komenda, T. Masopust, J. H. van Schuppen, Synthesis of controllable and normal sublanguages for discrete-event systems using a coordinator, Systems Control Lett. 60 (2011) 492-502.

[11] J. Komenda, T. Masopust, J. H. van Schuppen, Supervisory control synthesis of discrete-event systems using a coordination scheme, Automatica 48 (2012) 247-254.

[12] G. Jirásková, T. Masopust, State complexity of projected languages, in: Proc. of DCFS 2011, volume 6808 of Lecture Notes in Computer Science, Springer, 2011, pp. 198-211.

[13] C. G. Cassandras, S. Lafortune, Introduction to discrete event systems, Springer, 2nd edition, 2008.

[14] A. Salomaa, Formal languages, Academic Press, New York, 1973.

[15] W. M. Wonham, Supervisory control of discrete-event systems, 2011. Lecture notes, Department of electrical and computer engineering, University of Toronto. [Online]. Available: http://www.control.utoronto.ca/DES/.

[16] L. Feng, Computationally Efficient Supervisor Design for Discrete-Event Systems, Ph.D. thesis, University of Toronto, 2007.

[17] L. Feng, W. M. Wonham, Computationally efficient supervisor design: Abstraction and modularity, in: Proc. of WODES 2006, Ann Arbor, USA, pp. 3-8.

[18] J. Komenda, T. Masopust, J. H. van Schuppen, Synthesis of safe sublanguages satisfying global specification using coordination scheme for discrete-event systems, in: Proc. of WODES 2010, pp. 436-441.

[19] M. Sipser, Introduction to the theory of computation, PWS Publishing Company, Boston, 1997.

[20] T. H. Cormen, C. E. Leiserson, R. L. Rivest, C. Stein, Introduction to Algorithms, The MIT Press, 3rd edition, 2009.

[21] G. Karakostas, R. J. Lipton, A. Viglas, On the complexity of intersecting finite state automata and NL versus NP, Theoret. Comput. Sci. 302 (2003) 257-274.

[22] K. Wong, On the complexity of projections of discrete-event systems, in: Proc. of WODES 1998, Cagliari, Italy, pp. $201-206$.

[23] T. Moor, et al., libFAUDES - discrete event systems library, February 2012. [Online]. Available: http://www.rt.eei.uni-erlangen.de/FGdes/faudes/index.html.

[24] J. Komenda, T. Masopust, J. H. van Schuppen, Coordination control of discrete-event systems revisited, Discrete Event Dynamic Systems: Theory and Applications (2014). To appear, DOI: 10.1007/s10626-013-0179-x.

[25] P. N. Pena, J. E. R. Cury, S. Lafortune, Verification of nonconflict of supervisors using abstractions, IEEE Trans. Automat. Control 54 (2009) $2803-2815$

[26] H. Flordal, R. Malik, Modular nonblocking verification using conflict equivalence, in: Proc. of WODES 2006, pp. 100-106.

[27] H. Flordal, R. Malik, Compositional verification in supervisory control, SIAM J. Control Optim. 48 (2009) 1914-1938.

[28] R. E. Bryant, Symbolic boolean manipulation with ordered binary-decision diagrams, ACM Comput. Surv. 24 (1992) $293-318$.

[29] C. Ma, W. M. Wonham, Nonblocking Supervisory Control of State Tree Structures, Lecture Notes in Control and Information Sciences, Springer, 2005.

[30] T. Masopust, S. L. Ricker, Another approach to conditional decomposability for discrete-event systems, 2012. Manuscript. 